\title{
The cause of chronic prostatitis and prostate enlargement is a change in the vascular level and requires similar treatment: innovative Thermobalancing therapy
}

\author{
Allen Simon ${ }^{\star \dagger}$ \\ MD, PhD, Director, Fine Treatment, United Kingdom \\ DOI: https://doi.org/10.15520/ijmhs.v9i2.2453
}

Accepted 20 Feb 2019; Received 28 Jan 2018; Publish Online 28 Feb 2019

Reviewed By: Dr.

Daniel V.

Department: Medical

\begin{abstract}
INTRODUCTION: The outcomes of clinical studies show Thermobalancing therapy (TT) with Dr Allen's Therapeutic Device (DATD) successfully helps men treat chronic prostatitis / chronic pelvic pain syndrome (CP/CPPS) and lower urinary tract symptoms (LUTS) due to benign prostatic hyperplasia (BPH). The purpose of this study is to compare the ratio of the causes of these diseases with their treatment.

METHODS: Two clinical trials observed dynamics of urinary symptoms, pain score and prostate volume in men with BPH and CP/CPPS after using the device for six months. There were 124 men with $\mathrm{BPH}$ and 45 men with chronic prostatitis investigated in the treatment groups and the same number of men were in the control groups.

RESULTS: These studies have shown that TT with DATD reduced prostate volume from $45 \mathrm{~mL}$ to $31 \mathrm{~mL}(P<0.001)$ and, consequently, urinary symptoms from 14.2 to 4.9 $(P<0.001)$ in the treatment group of 124 patients with BPH. Pain score was reduced from 10.3 to $3.5(P<0.001)$ and prostate volume decreased from $31 \mathrm{~mL}$ to $27 \mathrm{~mL}$ $(P<0.001)$ in the treatment group of 45 men with $\mathrm{CP} / \mathrm{CPPS}$. In the control groups of 124 men with BPH and 45 men with $\mathrm{CP} / \mathrm{CPPS}$ no difference in the symptoms and parameters was observed.

CONCLUSION: The clinical studies indicated that the cause of these chronic diseases lies at the vascular level, namely the pathological activity of capillaries. The focus of hypothermia in combination with the spontaneous expansion of capillaries creates pressure in the tissue of the prostate gland, causing inflammation in it as well as symptoms in men with $\mathrm{CP} / \mathrm{CPPS}$ and prostate growth in men with BPH. TT with DATD proved to be effective in treating LUTS related to BPH and CP/CPPS. Therefore, the same therapy can be used for the treatment of both chronic prostatic diseases: $\mathrm{BPH}$ and $\mathrm{CP} / \mathrm{CPPS}$.
\end{abstract}

Keywords: cause of disease, Thermobalancing therapy; enlarged prostate; chronic prostatitis, prostate treatment

\section{INTRODUCTION}

\section{Prevalence}

Benign prostatic hyperplasia (BPH) is most often encountered in men over the age of 50 and can cause lower urinary tract symptoms (LUTS) [1]. Chronic prostatitis/chronic pelvic pain syndrome (CP/CPPS) is a common, painful disease in men under 50 . However, there is no useful

* Corresponding author.

$\dagger$ Email: info(at)finetreatment.com. treatment for this problem due to the unknown cause of the disease $[2,3]$.

The cause, etiology and pathophysiology of $\mathrm{BPH}$ and $\mathrm{CP} / \mathrm{CPPS}$

Discovering the cause of prostate enlargement is crucial for $\mathrm{BPH}$ treatment. It was thought that hormones have a fundamental role in BPH/LUTS development, particularly that androgens must be present for BPH to occur [4]. However, castrated boys do not develop BPH as they get older [5], Some years ago, investigations of BPH pathogen- 
esis focused on vascular dysfunction. For instance, aging could activate risk factors for systemic vascular disease, resulting in disturbed blood flow [6] . Development of prostatic hyperplasia could be associated with prostatic hypoxia [7]. Also, a correlation between pelvic ischemia and LUTS in elderly males [8], and increased pressure in the prostate gland have been suggested [9] .

The investigations on TT with DATD explored the etiology and pathophysiology of BPH and CP/CPPS, which can be explained by changes in small blood vessels in the affected tissues, namely the pathological activity of capillaries. In response to initial triggers-such as cold, infection, stress and other factors, the constriction of capillaries follows and that creates the focus of hypothermia, which is the secondary irritating trigger, thus leading to spontaneous expansion of a capillary net. Slowly this expansion of capillaries forms extra tissue in the prostate, which leads to prostate enlargement - BPH and LUTS [10] ; or creates pressure and prostate inflammation - CP/CPPS with associated symptoms [11] , including chronic pelvic pain [12] . This pressure inside the prostate tissue is responsible for the continuous compression of small vessels, which causes secondary local hypothermia and makes the problem chronic. This understanding of the pathophysiology of $\mathrm{BPH}$ and $\mathrm{CP} / \mathrm{CPPS}$ is a core principle of TT [13]

\section{Standard treatment options of $\mathrm{BPH}$ and $C P / C P P S$}

Antibiotics, medications and alternative strategies for $\mathrm{CP} / \mathrm{CPPS}$ are the subject of serious analysis as none of these treatment options are effective [14] . Medical treatments for $\mathrm{BPH}$ do not treat the prostate gland and have side effects negatively affecting the quality of life (QoL). Alpha blockers relax the bladder muscles, making it easier to urinate but cause tiredness, headaches, dizziness, retrograde ejaculation and others. Another class of drugs, 5-alpha reductase inhibitors, such as finasteride and dutasteride, act at the hormonal level to ease urination - but cause impotence, depression, testicle pain, and even diabetes. [15]

\section{TT with DATD for $B P H$ and $C P / C P P S$}

TT with DATD was developed for the treatment of chronic internal diseases by using the body's own energy. [16] . This therapy has demonstrated pain relief and improvement of QoL in men with CP/CPPS [17]. TT with DATD diminishes LUTS due to BPH by reducing the enlarged prostate volume [18]. This article is intended to show that TT with DATD is able to treat the cause of BPH and $\mathrm{CP} / \mathrm{CPPS}$ in the same way.

\section{METHODOLOGY}

Dr Allen's Device was registered with the Medicines and Healthcare Products Regulatory Agency in the United Kingdom in 2010, as a class 1 medical device. The ethics committee of the Yerevan State Medical University approved the clinical study with TT and DATD. The studies were registered at the World Health Organisation via the German Clinical Trials Register (DRKS). TT with DATD was used in 2 clinical controlled studies at the Department of Urology of the Yerevan State Medical University. One clinical trial in 124 men with BPH who received TT within a 6-month period examined their clinical parameters before and after therapy. This information was compared with the control group, i.e. data received from 124 men with BPH who were in watchful waiting. The second clinical trial of 45 men with CP/CPPS who received TT within a 6-month period examined their clinical parameters before and after therapy. This information was compared with the control group, i.e. data received from 45 men with CP/CPPS who did not receive TT. Patients with BPH were measured using the International Prostate Symptom Score - Quality of Life (I-PSS). In men with CP/CPPS the National Institute of Health Chronic Prostatitis Symptom Index (NIH-CPSI) score was utilized. In both clinical studies, prostate volume (PV $\mathrm{mL}$ ) was investigated. The parameters were compared between groups accordingly. Baseline evaluations were a full physical examination, medical history, digital rectal examination, serum biochemistry, measurement of prostatespecific antigen and electrolytes, urinalysis, and renal function tests. Dynamics of the symptoms and indicators in each group were assessed at the beginning and end of treatment using NIH-CPSI and I-PPS scores. Ultrasound was used to determine the volume of the prostate gland $(\mathrm{PV} \mathrm{mL})$. The independent-samples t-test and paired-samples t-test are suitable only for interval and ratio data, so the Wilcoxon signed-rank test was employed. $\mathrm{P}<0.05$ was considered significant. Statistical analyses were carried out using SPSS v22 (IBM, Armonk, NY, USA).

\section{$D A T D$}

DATD applies a special mixture of waxes (thermoelement) topically to be projected toward the affected organ. In men with prostate problems, it would be directed to the coccyx area Figure 1. The thermoelement accumulates the emitted body heat and turns into a source of energy itself. DATD applies the thermoelement tightly to the skin, thereby overcoming the skin barrier and spreading the energy towards the prostate gland.

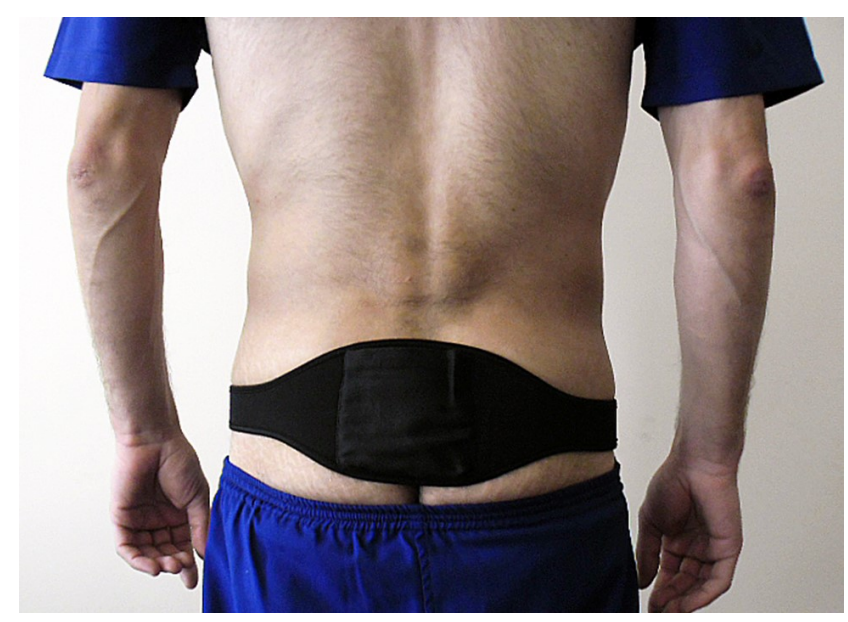

Figure 1. DATD tightly attaches a thermoelement to the coccyx area in a man for prostate treatment. 


\section{RESULTS}

Figure 2 Dynamics of prostate volume (PV) mL, measured by ultrasound, and pain score in 45 men withCP/CPPS, measured by the National Institute of Health-Chronic Prostatitis Symptom Index (NIH-CPSI), in 45 men withCP/CPPS and in the control group.

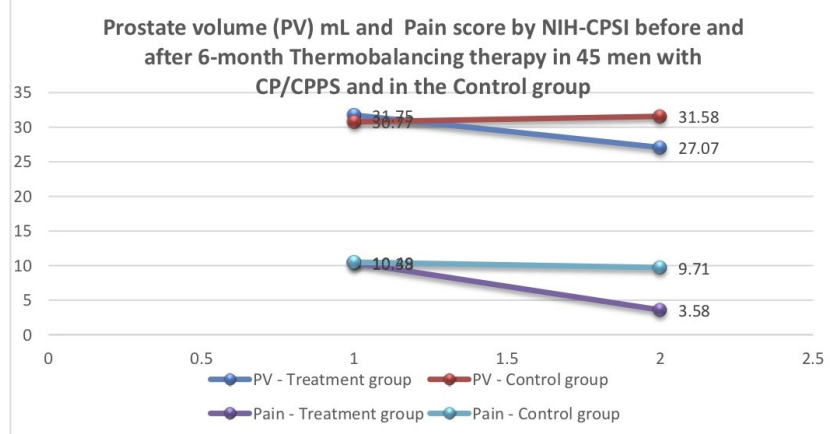

Figure 2. As indicated from this figure there was a significant decrease of PV $(\mathrm{mL})$ from $31.75 \pm 7.0$ to 27.07 $\pm 4.5 \mathrm{~mL}(P<0.001)$ and pain score from $10.38 \pm 2.53$ to $3.58 \pm 2.54(P<0.001)$ in the treatment group. In the control group changes were insignificant. These results suggested that TT decreases $P V$ and pain in men with CP/CPPS.

Figure 3 Prostate volume (PV) mL, measured by ultrasound, and urination symptoms (UrS), measured by the International Prostate Symptom Score (IPSS), in 124 men with BPH after 6-month Thermobalancing therapy, and in the control group.

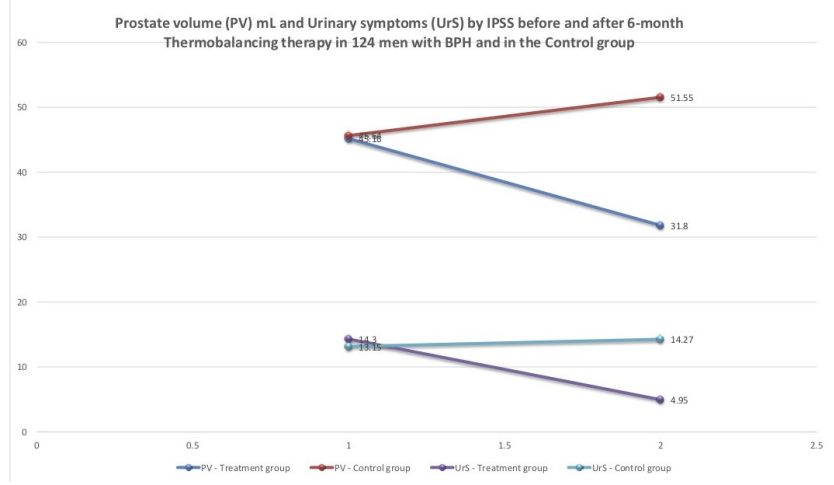

Figure 3. According to this figure, in the treatment group PV decreased from $45.1 \mathrm{~mL}$ to $31.8 \mathrm{~mL}(P<0.001)$ and UrS score decreased from 14.3 to $4.9(P<0.001)$. In the control group changes were insignificant or even worse. These results suggest that DATD reduces $P V$ and UrS significantly.

\section{Side effects of Thermobalancing therapy with} DATD

Side effects of Thermobalancing therapy with DATD were not observed.

\section{DISCUSSTON}

The use of TT with DATD reduces volume of the inflamed prostate and pain score in patients with CP/CPPS dramatically, whereas in the control group changes were insignificant [19] . In men with BPH the size of enlarged prostate has been reduced greatly and, consequently, LUTS relief has been achieved [20]. At the same time, the prostate size in men with $\mathrm{BPH}$ in the control group increased and urinary symptoms worsened.

The importance of these studies also includes the high costs of CP/CPPS and BPH treatments. For instance, the annual per person costs of treatment of a man with CP/CPPS were estimated as $\$ 6534$ [21] ; and annual cost of BPH medical treatment was lowest in the UK at about $\$ 1000$ and highest in Poland at approximately $\$ 1500$ [22] . The use of TT with DATD, with the price for a unit less than \$200, would make significant changes in the costs of these common chronic diseases.

The studies have proven that TT with DATD can be easily implemented in the medical practice in the primary health care system [23]. The use of DADT which redirects energy accumulated from the body in the projection of the prostate gland for a prolonged period, has deactivated the micro-focus of hypothermia and spontaneous expansion of capillaries, thereby relieving pressure in the prostate tissue and reducing the enlarged or inflamed prostate in size [24] .

Although $\mathrm{BPH}$ and $\mathrm{CP} / \mathrm{CPPS}$ have nasty symptoms, they are non-life-threatening conditions. Therefore, these chronic internal diseases should be treated with safe TT as first line therapy to aid in maintaining men's wellbeing $[25,26]$. It should be noted that Thermobalancing therapy treats the cause of CP/CPPS and BPH by improving blood circulation in the prostate gland in similar ways.

\section{CONCLUSION}

TT with DATD is an economical solution for chronic prostate disease management, as the price is cost-effective compared to other treatment options. TT with DATD terminates the pathological activity of capillaries and enhances blood circulation in the affected prostate, thus treating the cause of BPH and CP/CPPS. DATD provides the same safe treatment using our own human body energy for $\mathrm{BPH}$ and CP/CPPS identically. The use of TT with DATD for the treatment of $\mathrm{BPH}$ and $\mathrm{CP} / \mathrm{CPPS}$ can significantly improve men's quality of life and reduce pressure on the healthcare system.

\section{Competing interests}

These studies were not supported by grant and did not have financial interest.

\section{ACKNOWLEDGMENTS}

I am grateful to Professor IG Aghajanyan, the founder of the Armenian Association of Urology for his support in conducting clinical studies. 


\section{REFERENCES}

[1] Rohrmann S, Katzke V, Kaaks R. Prevalence and Progression of Lower Urinary Tract Symptoms in an Aging Population; 2016.

[2] Abdollahi A, Etemadian M, Shoar S, Nozarian Z. Is Helicobacter Pylori Infection a Risk Factor for Prostatitis? A Case-Control Study in a Referring Tertiary. Care Center, Iran J Pathol;2016:11-4.

[3] Disord. Chung SD, Huang CC, Lin HC Chronic prostatitis and depressive disorder: a three-year population-based study. J Affect. 2011;

[4] S MB. RP et al, Managing the Risks of Prostate Disease During Testosterone Replacement Therapy in Older Men: Recommendations for a Standardized Monitoring Plan. Journal of Andrology;2003(24):299-311.

[5] Wu CP, Gu FL, The prostate in eunuchs, Prog Clin Biol Res;

[6] S STS. et al, Lower urinary tract symptoms, benign prostatic hyperplasia/benign prostatic enlargement and erectile dysfunction: Are these conditions related to vascular dysfunction? Int J Urol. 2014 9;9(856-64).

[7] M OS. R et al Prostatic ischemia induces ventral prostatic

[8] P T. Yang JH, Azadzoi KM LUTS in pelvic ischemia: a new concept in voiding dysfunction. Am J Physiol Renal. 2016 1;20:00333-2015.

[9] Cohen PG, Abdominal obesity and intra-abdominal pressure: a new paradigm for the pathogenesis of the hypogonadal-obesity-BPH-LUTS connection; 2012.

[10] S A. Aghajanyan IG. Use of thermobalancing therapy in ageing male with benign prostatic hyperplasia with a focus on etiology and pathophysiology, Aging. Male;2016(1-5):101080.

[11] S A. The Cause, Aetiology and Physiopathology of Chronic Prostatitis/Chronic Pelvic Pain Syndrome (CP/CPPS) and its Effective Treatment with Thermobalancing Therapy. Med Sur. Urol;2018(7):10-4172.

[12] Allen S. The Vascular Factor Plays the Main Role in the Cause of Pain in Men with Chronic Prostatitis and Chronic Pelvic Pain Syndrome: The Results of Clinical Trial on Thermobalancing Therapy. Diseases;2017(5):25.

[13] Allen S. The Origin of Chronic Diseases Can Be in Capillary Pathology: An Evidence from Clinical Trials on Thermobalancing Treatment of Prostate Reveals; 2016.

[14] Pharmacological interventions for treating chronic prostatitis/chronic pelvic pain syndrome (Protocol). Cochrane Database of Systematic Reviews 2017, Issue 2. Art; 125.

[15] Benign prostatic hyperplasia treatment options and their effects on sexual function. Sex Med Rev;5(87-102):10-1016.

[16] S AA. Therapeutic Device and Method, United States Patent and Trademark Office. US Patent. 2016 $8 ; 9(408)$. Available from: https://www.google.com/patents/ US9408744.

[17] S A. Thermobalancing therapy as Self-management tool. vol. 2019;

[18] S A. Aghajanyan IG. Thermobalancing conservative treatment for moderate - to - low - degree lower urinary tract symptoms (LUTS) secondary to prostate enlargement. Cogent Medicine;2016(3):1195067.

[19] Allen S, Aghajanyan IG. Effect of thermobalancing therapy on chronic prostatitis and chronic pelvic pain syndrome. Journal of Clinical Urology. 2016 9;20:1-8.

[20] S A. Aghajanyan IG. Benign Prostatic Hyperplasia Treatment with New Physiotherapeutic Device.Urol J;2015:14-12
[21] Clemens JQ, Markossian T, Calhoun EA. Comparison of the economic impact of chronic prostatitis/chronic pelvic pain syndrome and interstitial cystitis/painful bladder syndrome. Urology;

[22] Burden of male lower urinary tract symptoms (LUTS) suggestive of benign prostatic hyperplasia $(\mathrm{BPH})$ - focus on the UK. BJU Int;2015(115):508-519.

[23] Dr AS. Allen's Therapeutic Devices Should be Implemented in the Healthcare System for the Treatment of Chronic Noncancerous Prostate and Kidney Diseases Saving People's Well-Being and Money. Ann Mil Health Sci Res;2018(16):81033.

[24] IG ASA. Positive Response to Thermobalancing Therapy Enabled by Therapeutic Device in Men with Non-Malignant Prostate Diseases. vol. 2016;.

[25] Mon. Allen S, Aghajanyan IG New independent thermobalancing treatment with therapeutic device for internal diseases: chronic prostatitis and chronic pelvic pain syndrome, Nephrourol; 2017.

[26] S A. Innovative Thermobalancing therapy can help millions of men with enlarged prostate gland to improve the quality of life and well-being throughout the world;

\section{AUTHOR BIOGRAPHY}

Allen Simon MD, PhD,

Director, Fine Treatment, United Kingdom 